\title{
Register of persons and places
}

Aarhus 419, 433

Adolf, Duke of Holstein-Gottorp 118, 251, 421

Adriansen, Johan 214

Africa 13

Aith 226

Aithsting 164, 184

Akranes 109, 209, 215, 259-262, 460

Akurey 257

Akureyri 47, 209, 292

Albert de bartscherer 177

Álftafjörður 109, 112, 189, 214, 215, 284-288, 369, 419, 421, 435

Alberts, Heinrich 278, 433, 436, 477

Amsterdam 30, 33, 53, 85, 97, 102, 242, 310, $349,419,420$

Amund, Bishop of the Faroe Islands 127, 197

Andersen, Peder 435

Andersen, Søren 435, 441

Anteman, Johan 487

Anthony I, Count of Oldenburg 117, 333, 417

Anthony II, Count of Oldenburg 333

Antvorskov 421, 430

Antwerp 53, 74, 242, 247, 347, 349

Arnarfjördur 281, 282, 284

Arnarnesheiði 173

Arnarstapi 109, 113, 135, 146, 159, 192, 194, 209, 262-269, 272, 341, 368, 371, 374, 418, 421-424, 426, 428, 430, 436, 451, 454, 456, 461-462, 464-465, 473-474, $478,480,486$

Arndes, Johan 444

Arngrímur Jónsson 13, 14, 16, 141, 174

Árni Oddsson 193

Arres, Peter 134

Austurfjörutangi 304

Averie, Joshua 184, 316

Backmeister, Lutke 444

Bade, Berendt 444

Bade, Otto 209, 292

Baelen, Cordt van 487

Bagge, Ólafur 183, 188
Bake, Carsten 113, 143, 148, 162, 192, 193 , $258,259,275,277-281,342,353,376$, 423, 427, 428, 432, 478

Bake, Vasmer 135, 144, 262, 478

Baleke, Paul 444

Baleman, Hans 444

Balleers, Johan 487

Ballentyne of Garsay, William 184

Baltasound 215, 487-492

Baltic Sea 52, 63, 66, 154, 218, 329, 330, 383

Baltzer de bartscherre 177

Baltzerssen, Matz 195

Barby, Andres von 103

Barchstede, Michael 119, 293

Bardaströnd 113, 179, 183, 279, 281-286, $341,342,351,353,424,429,431,436$, $451,454,459,472$

Bare, Margarete 368

Barnefeld, Paul 245, 364, 431, 437, 444

Barnewolt, Evert 487

Bartels, Johan 444

Bartscherer, Henninck 177

Bartscherer, Hinrick 177

Bartscherer, Jacob 176

Bartscherer, Johan 487

Básendar 43, 75, 79, 109, 113, 119, 140, 141, $176,177,209,214,215,243,244,246$, 247, 248, 249, 252, 254, 258, 370, 418, 419, 420, 422, 423, 425, 429, 431, 433, 437, 445, 448, 454, 455, 461, 462, 464-467, 474-476

Basse, Cordt 192, 294, 295, 297, 345, 360, $361,427,430,432,435,444$

Basse, Jochim 445

Becker, Buske 445

Beling, Arendt 487

Beling, Johan 134, 487

Bene, Carsten 445

Berchman, Jacob 177

Bergen 3, 5, 11, 20, 22, 23, 27, 30, 35-37, $39,40,45,47,50,52,53,59,61,64$,

¿ Open Access. (C) 2020 Bart Holterman, published by De Gruyter. (cc) BY-NC-ND This work is licensed under a Creative Commons Attribution-NonCommercial-NoDerivatives 4.0 International License.

https://doi.org/10.1515/9783110655575-017 
66-72, 76-79, 81-92, 94, 97, 98, 124-129, 132, 133, 141, 143, 149, 154, $155,157,158,161,163,171,179,180$, $183,185,190,195,197,208,216,221$, $233,267,310,315,323,325,327,328$, 359, 365, 377-379, 438, 439, 440, 491

Berman, Dirick 360, 435, 438

Berman, Hans 192, 203, 346, 445

Berman, Henning 445

Berman, Wichman 346, 445

Berndes, Hinrick 177

Berufjördur 93, 109, 111, 113-115, 147, 148, $158,159,161,168-170,176,181,188$, $189,199,209,211,212,215,223,225$, 229, 242, 296-305, 334, 338, 357, $360-363,366,375,419-421,427$, 429, 430, 432, 433, 435, 437, 451, 478-486

Bessastadir 99, 173, 183, 186, 191, 239, 254 Betke, Mauritz 445

Betke, Peter 445

Beverborch, Herman 267, 271, 336, 355, 446

Bicker, Arp 487

Biedendorf, Jurgen 447

Bigton 218

Bilde, Eski 126, 127, 439

Bíldudalsvogur 284

Bíldudalur 109, 215, 282-284, 286, 418

Binneman, Reineke 487

Bisterfeld, Daniel 303

Bjarnarhöfn 271

Bjarni Eiríksson 173

Björn Gunnarsson 189

Björn Jónsson 183, 280, 432, 480

Björn Porleifsson 72, 190

Blacater, John 134

Blaeu, Willem 218, 220

Blak, George 134

Blefken, Dithmar 2, 13, 14, 16, 46, 53-55, $139,151,172,178,223$

Bleke, Cordt 438, 446

Blome, Cordt 13, 53, 54, 107, 172, 175, 446

Blome, Hans 446

Blome, Hinrick 487

Bockenheim, Jean of 29
Bockholt, Johan 173, 188, 189, 256, 273 , $302,353,357,422$

Boddeker, Hans 173

Bode, Hinrich 368

Boleman, Bernt 478

Bollandt, Cordt 345

Bolungarvík 284

Bomhofer, Gert 283, 418

Borchers, Jurgen 262, 264

Borgarfjarðarsýsla 261

Borgarfjördur 297

Bornholm 97

Bornhorst, Christoffer 478

Börringe 418

Borstel, Albert vam 446

Borstel, Ratke vam 446

Boske, Boske 478

Boston 66, 68, 72, 143

Bothnian Gulf 223

Botker, Cordt 296, 297, 366, 424, 446, 461

Botman, Jochim 176

Bramstede, Johannes 321

Brand, John 226, 229

Brandt, Dirich 447

Brandt, Franz 53, 165, 360, 447

Braunschweig 84, 343, 358

Brede, Eler 324, 335, 487

Breiðafjörður 202, 229, 267, 268, 276, 279. 281, 282

Breker, Gerdt 52, 150, 196, 216, 224, 356, 487, 489

Bremen 1-3, 6, 7, 9, 11, 15, 16, 18, 22, $30-33,36,40,42-45,52,53,55-57$, $66,68,72,75,77,82-84,86-93,104$, 110-120, 132-136, 138, 139, 141-144, $146,147,149,150,153,158,159,161$, $162,164,166-170,173,174,176,180$, $181,183,184,186,188,189,192,193$, 195, 199, 200, 202-204, 209, 211-213, 215, 216, 219, 220, 222-227, 229-232, 236, 242, 244, 245, 249, 250-252, 255, 258, 259, 262-281, 295-305, 311, 316, 323-327, 332-335, 338-343, 346, 347, 351-363, 366-369, 374-376, 378, 382, 
383, 419-421, 423, 427-430, 432, 433, 435-437

Bremen, Archdiocese 32, 61, 116, 357

Bremer, Johan 164

Brender, Jacob 435

Bressay 218, 226

Bressay Sound 216, 218, 487, 491

Breye, John 75, 145, 244

Brinckman, Carsten 487

Bristol 70

Brockman, Hinrich 478

Brockman, Jost 362, 478

Bruce, Lawrence 148, 149, 196, 231

Bruce of Symbister, William 184, 231

Bruges 67, 68, 79, 80, 89

Brummer, Hinrick 487

Brummer, Luder 184, 487, 491

Bruns, Herman 176

Bruns, Wichman 180, 488

Brunswick-Lüneburg, Duchy 263

Buchanan, George 57

Buckhoren, Johan 488

Buer, Jochim 447

Búdanes 275

Búdaós 261, 262

Búdasandur 228, 260

Búdir 111, 113, 135, 144, 146, 149, 159, 192, 209, 242, 261-265, 272, 418, 419, 427, $430,436,456,478,480,482,483,485$

Buerman, Herman 478

Buneke, Hans 177, 209, 292, 447

Buramer, Bertolt 81

Burgundy 54

Burhorn, Jost 447

Burra 218

Burravoe 48, 184, 216, 488, 491

Busing, Jasper 225

Busk, Niels 252, 281

Busta Voe 229

Buxtehude 112, 113, 251, 342, 360, 421

Bygholm 419

Campe, Hans van 448

Campe, Jasper van 448

Carstens, Albert 176
Carstens, Carsten 448

Chalmer, Robert 134

Chester 135, 358

Christian I, King of Denmark 3, 73, 84, 85, 93, 94, 378

Christian II, King of Denmark 73, 88, 97, 98, 125, 126

Christian III, King of Denmark 98, 100-103, 118, 132, 181, 255, 349, 417

Christian IV, King of Denmark 3, 116, 119-123, 163, 227, 228, 234, 248, 378

Clawes de bartscherer 177

Clerck, Thomas 135

Cogill, William 44

Collen, Lucas van 320,321

Cologne 30, 66

Cologne, Archdiocese 33

Copenhagen 7, 47, 64, 76, 88, 90, 92, 100-105, 109, 111, 112, 120, 122, 123 , $128,129,130,132,174,175,180,183$, 192, 195, 198, 207, 239, 243, 246, 248, 251, 255, 256, 257, 260, 264, 281, 283, 291-293, 296, 300, 303, 348, 349, $354,378,417-423,426-436,438$, 440, 441

Cordes, Harmen 143, 338

Cordes, Johan 215, 216, 488

Cordes, Matthias 244

Cordes, Wilcken 209, 360

Cörver, Jacob 448

Cracow 47

Craxton, John 290

Culla Voe 220

Cullivoe 134, 216, 490

Daberhol, Heyne 448

Dambeke, Peter 80, 143, 171

Daniel de bartscherer 177

Danninck, Jochim 364, 448

Danzig 7, 72, 80, 83-85, 87, 104, 124, 143-145, 171, 347, 349, 377

Davorde, Hinrick 373, 448

Daye, Thomas 28, 143, 342

Debes, Lucas 221

Delmenhorst, Anna 225, 258, 352 
Delmenhorst, Hans 135, 144, 225, 258, 259 , $327,351,352,358,423$

Denmark 3, 5, 7, 10, 15, 16, 33, 43, 46, 47, $50,52-54,64,71-75,79,81,82,84,87$, 90-95, 97-103, 105- 107, 109-114, $116-122,124,125,127-133,135,139$, $143,145,161,162,167,171,174,180$, 183, 185, 187, 190, 195, 210, 211, 221, 227, 234, 237-240, 242-244, 246, 248, 250, 252-254, 256, 257, 259-261, 264-269, 271, 272, 274, 279-282, 284-290, 292, 293, 295, 296, 298, 303, 304, 315, 321, 340, 344, 346, 348-351, 354, 356, 357, 361, 369, 371, 377-380, 382-384, 432

Detken, Gerdt 200, 204

Detken, Hendrick 225, 232

Detken, Herman 164, 165, 200, 204, 225 , 488

Detken, Magnus 164, 488

Detken, Segebad 1-3, 134, 184, 203, 215 , 216, 231, 362, 376, 487-489, 491

Detlefs, Carsten 448

Detlefs, Clawes 448

Detlefs, Reymer 448

Detzelman, Cordt 345

Deventer 30, 88, 132

Deventer, Rulf von 119

Dickmeyer, Reymer 448

Djúpivogur 212, 299, 303, 427, 432

Doerman, Hans 448

Doren, Hans van 352

Doren, Heinrich van 352

Doren, Jasper van 259, 336, 352, 437, 449

Doren, Marx van 448

Dorothea, Queen consort of Denmark 109, 116

Dose, Heinrich 342

Double, Cornelius 125

Dranteman, Clawes 478

Drape, Hans 367, 449

Drape, Jurgen 449

Dreyer, Gerdt 478

Dunrossness 164, 216, 218, 219, 490, 492

Durkop, Radtke 479
Dýrafjördur 93, 109, 110, 183, 215, 281, 284-286, 328, 348, 349, 369, 418-420, 422

East Anglia 70

East Frisia 13, 88, 273

Eastfjords 189, 211, 295, 297, 298, 380

Ebeling, Otto 449, 450

Eckhoff, Peter 177

Eddelman, Johan 479

Edinburgh 8, 184

Eding, Hans 450

Eding, Lutke 450

Edmistoun, James 134

Edward IV, King of England 72

Egeler, Berendt 479

Egendorf, Johan 435

Eggers, Clawes 450

Eggers, Hans 291, 346, 366, 373, 432, 436, 450

Eggers, Hinrick 489

Eggers, Jacob 450

Eggers, Matthias 144, 159, 188, 291, 296, $302,347,366,367,424,429,431,450$

Eggert Hannesson 46, 173, 179, 183, 188, 189, 208, 209, 241, 244, 258, 263, 269, $283,284,286,363,422$

Einarshöfn 240

Eiríkur Árnason 159, 181, 182, 188, 189, 192 , 224, 300, 302

Eitzen, Meinert von 175

Eiðisvík 256

Elbe $89,112,123,144,145,175,248,256$, $258,259,291,341,366,441,450$

Elers, Barteld 287, 345, 352, 425, 428, 431, 433, 436, 451

Elers, Daniel 302-304, 338, 347, 348, 360, $361,427,432,434,451$

Elers, Hans 289, 346, 360

Elers, Johan 452

Elers, Jurgen 113, 451

Ellebracht, Johan 487-492

Elmshorn 50

Embdeman, Grete 492

Emden 134, 274, 315, 349 
Engelken, Hans 452

Engelken, Werner 452

England 15, 17, 28, 30, 31, 39-41, 45, 47, 50, $53,54,64,66,67,69-77,79-85$, $87-91,93,95,97,98,100,101,109$, $123,130,133,135,136,140,141$, $143-145,157,159,163,172,186,187$, $189,190,198,210,224,238,239,243$, 244, 247, 249, 251, 253-255, 258, 259, 265, 266, 269, 272, 285, 287, 290, 292, 293, 304, 310-316, 323, 346, 347, 351, $358,359,370,373,377,378,381,443$

Erasmusdochter, Margarete 180

Erfurt 30, 320

Eric of Pomerania, King of Denmark 64, 79, 81

Ericksen, Klaus 281, 433

Erlendur Magnússon 189

Erlendur Porvardarson 368

Ertman, Herman 164

Esick, Hinrick 325, 489

Eybe, Peter 452

Eyjafjördur 47, 75, 176, 209, 228, 288, 290-295, 304, 320, 338, 420, 435, 437, 448, 454, 456, 458, 468, 469, 473, 475

Eyrarbakki 57, 113, 197, 198, 209, 239-243, $245,246,253,254,342,345,349,351$, $352,354,419,420,422,425,429,435$, 446-448, 453, 456, 462, 468, 472, 474, 475

Eyrarsveit 159

Eys, Gertrud 368

Eys, Roleff 203, 236, 287, 341, 368, 431, 433, 451

Eysturoy 129

Fabricius, David 13, 14, 141, 151

Fabritius, Johan 321

Falckner, Johan Jellesen 33, 242, 349, 350, 419, 420

Falenkamp, Berent 479

Faroe Islands $3-6,8,9,16,17,19,33,35$, $40,44,48,52,53,55,57,59,62,63,69$, $78,79,82,83,87,88,90,97-99,111$,
117, 119, 124-133, 135, 141-144, 148, $149,155,163,164,175,180,181,183$, 194, 195, 197, 201, 208, 221, 222, 226, 233, 236, 322, 336, 338, 345, 347, 348, $349,350,354,360,361,368,370,371$, 373, 377-380, 382, 383, 439, 441, 443, 444, 451, 471, 475, 476

Faxaflói 253

Felthusen, Berent 489

Fetlar 164, 165, 184, 216, 221

Ficken, Clawes 9, 363, 479

Fincke, Jacob 304, 435

Finland 80

Finnmark 80

Flanders 54, 310, 311, 329, 347, 373

Flatey 113, 183, 193, 211, 267, 273, 279, 280, $281,283,353,422,423,427,428,432$, $433,435,438,467,478,480$

Flege, Jurgen 452

Flensburg 103, 113, 119, 187, 304, 435

Focke, Joachim 189, 192, 294, 295, 297, 298, 302, 304, 305, 424, 426, 429, 431, 434, 452

Foggo, George 134

Folkers, Cordt 489

Fothe, Marcus 176

Fowler, John 50

Fox, Frederich 177

France 42, 55, 133, 268, 321, 359

Francke, Johan 177

Frankfurt 320

Frederick I, King of Denmark 75, 97, 98, 119, 126, 127

Frederick II, Holy Roman Emperor 49

Frederick II, King of Denmark 47, 50, 53, 58, $92,103,104,105,107,109,111,112,116$, $118,119,128,130,264,277,288,292$, $348,349,350,378$

Frederiksborg 419, 420, 421, 422, 440

Frerick de tymmerman 228, 234

Frese, Alert 177

Frese, Berndt 177

Frese, Clawes 453

Frese, Folckert 251, 479

Frese, Hans 338, 453 
Frese, Meinert 177, 209

Frese, Richard 179

Fresenberg, Peder 126, 439

Frigdach, Carsten 453

Froudendal, Cordt 247

Fýluvogur 159, 212, 303, 433, 437

Gading, Johan 177

Gansberch, Herman 297

Garleffs, Heyn 453

Gásir 228, 292

Gautavík 57, 212, 228, 229, 298, 299

Gedde, Brostrup 193

Geirhólmur 286

Gelker, Cordt 321

Geners, Hinrick 176

Gerbade, Gerdt 361, 479, 489

Gerdes, Johan 173

Gerdes, Roleff 273, 332, 334, 340, 341, 353, 367,479

Gerdes, Thomas 342

Gerkens, Hannes 178, 453

Gerkens, Hans 178, 453

Gerkens, Henrik 177, 178, 453

Gersteman, Bartholomeus 200

Gettle, Henrick 50

Giamor, Robert 134

Giffert, William 134

Gisze, Georg 145

Gizur Einarsson 56, 100, 174, 198, 241

Gjerskov 440

Glückstadt 119, 123

Gluss 196, 218, 219

Godske, Andres 248, 418

Goldener, Peter 453

Goslar 47

Gotken, Carsten 50

Gotken, Heyn 50

Gotkens, Simon 453

Gotland 97

Gottorp 439

Grale, Peter 489

Grandahólmi 256, 257

Grascher, Wolder 479

Graskamp, Jurgen 454
Grasmoller, Hein 200

Grasmoller, Hinrich 143, 454

Great Britain 43

Greenland 13, 49, 53, 146

Greenland Strait 281

Greifswald 144

Grelle, Reineke 244

Greppel, Albert 177

Greve, Joachim 438

Griep, Joachim 179

Grindavík 75, 113, 117, 144, 187, 191, 209 , 243-249, 254, 256, 258, 375, 420, 425, 426, 428, 431, 433, 437, 444, 458, 465, 481, 482, 485

Gronewold, Hans 264, 266, 269, 341, 421, 454

Groper, Alberth 176

Groper, Dirick 209

Grove, Jochim 144

Grove, Jurgen 248, 425, 429, 454

Grundarfjörður 109, 113, 118, 199, 209, 262, 264, 266-272, 274, 275, 277, 336, 341, 422, 423, 433, 437, 446, 457

Grundarkampur 268

Grunnasundsnes 275

Grutness Voe 220

Grýluvogur 279

Guðbrandur Porláksson 199, 291, 422

Gudmunder Niclawessone 180

Guðmundur Pórðarson 188

Gudrun, daughter of Sigurd 180

Gufuskálar 266

Gunnewitt, Arendt 454

Gunnister Voe 196, 197, 218, 219, 231

Guttormsen, Anders 194

Gyldenstern, Magnus 104

Gyseke, Bernd 98

Haakon IV, King of Norway 63

Hackman, Johan 489

Haderslev 422, 434, 435

Hafnarey 279

Hafnarfjördur 16, 54, 74, 75, 85, 95, 99, 109, $111,113,118,121,141,148,150,158$, $160,161,173,175-178,183,186,187$, 
189, 191, 209, 213-215, 223, 227, 228, 234, 236, 241, 244, 246, 247, 249, 250, 252-256, 258-260, 283, 284, 296, 314, 317-321, 338, 340, 345, 348, 354, 357, $365,370,371,381,382,419,421,422$, 425, 430, 432, 434, 438, 444-449, 451-467, 471-475

Hagen, Arndt vam 244, 454

Hagen, Hans vam 454

Hagen, Johan 489

Hagen, Jurgen vam 176, 177, 255

Hagen, Michael vam 455

Háigrandi 234, 253, 254

Haithabu 37

Halmstad 94

Hamborch, Johan 81

Hambrock, Catharina 367

Hambrock, Dirick 438, 455

Hambrock, Hans 151, 357

Hambrock, Jacob 252, 367, 433, 435, 438, 455

Hamburg 1-7, 9-13, 15-18, 22, 30-32, 39, $40,42-48,50-56,58,66,68,72$, 74-77, 79, 83-93, 95-113, 115, 116, 118-136, 140-148, 150, 151, 153, 158-160, 162-165, 168, 170, 171, 173-176, 178-189, 191, 192, 195, 198-200, 202, 203, 209, 211, 214, 215, 219, 220, 221, 223, 225-228, 230, 231, 233, 235-237, 239, 241, 242, 244-255, 258-260, 262-264, 266-269, 271-273, 280, 283-287, 289-292, 294-298, 302-305, 308-316, 319-327, 331-338, 340-342, 344-347, 349, 350, 352-357, $359,360,362,363,365-370,372,373$, $375,377-383,417,419,421,423-440$, 443, 479

Hammeltong 134

Hamna Voe 220

Hane, Christoffer 321

Haneman, Hans 479

Haneman, Hinrick 269, 272

Hannes Eggertsson 187

Hannover 145

Hans de balberer 177
Hans de Barscheyrer 176

Hanssøn, Jørgen 125, 439

Harald Maddadson, Earl of Orkney 62

Harbarge, Hans 455

Harburg 31

Hardt, Hermen 456

Hare, Hans 144, 246, 456

Hare, Joachim 177, 364, 434, 435, 438, 456

Haroldswick 225, 232

Harriestede, Simon 164, 196, 197, 219, 221, 231, 456

Harst, Johan 480

Hartge, Johan 456

Hartich, Hans 296, 302, 456

Hartman, Christoffer 456

Harvest, Johan 271, 275, 336, 437, 457

Harwede, Cordt 209

Harwich 247

Hase, Hans 264, 267, 355, 429

Hasow, Hans 173

Hebrides 63

Hegewisch, Cordt 480

Heide, Cordt van der 489

Heihusen, Alert 346, 457

Heimaey 237

Heine, Marcus 105

Heinesen, Magnus 129, 130, 163, 183, 440

Heins, Hans 183

Heit, Jost 321

Heldtberch, Cordt 457

Helgafell 27, 47, 198, 203, 205, 268, 276, 482

Helgoland 144, 246

Hellissandur 266

Helsingør 120, 122, 135, 146, 214, 248, 264, 281, 284, 288, 421, 433

Hemeling, Cordt 52, 150, 196, 216, 224, 356, 487, 489, 492

Hemeling, Gerdt 133, 134, 150, 184, 196, 220, 325, 357, 376, 490

Hemeling, Johan 166, 490

Henninck de barscherer 177

Henry III, Archbishop of Bremen 32, 117, 269, 274, 277, 341, 423 
Henry Julius, Duke of Brunswick-

Lüneburg 374

Henry VIII, King of England 75

Hepburn, James, Earl of Bothwell 133, 134, 357, 490

Hering, Hans 192, 294, 295, 297, 345, 367, 427, 430, 432, 435, 438, 457

Hermansen, Reinicke 181

Hess, Marcus 248, 255, 264, 265, 267, 269 , 291, 292, 296, 349, 354, 419, 420, 421, 422

Hesse 29

Hesterberch, Anna 179

Hesterberch, Armgard 179

Hesterberch, Arndt 46, 179, 457

Hesterberch, Hans 314, 457

Hildesheim 190

Hillswick 218

Hilmers, Cordt 490

Hinck, Joachim 116, 375

Hinricks, Andreas 321

Hintzke, Hinrick 175, 198, 241, 457

Hjaltastaðir í Utmannasveit 159, 212

Hoeggers, Hinrick 490

Hoep, Matthias 50, 51, 53, 316

Hoffgaard, Hans 257

Hoffman, Andreas 321

Hofsós 110, 113, 199, 209, 290-292, 296, 418, 422, 424, 429, 431, 432, 436, 450, 452, 463

Hólar 71, 162, 197, 199-201, 290-292

Holbein, Hans 145

Holland 73, 85, 94, 126, 199, 253

Holler, Cordt 490

Holm (Pinneberg) 12

Hólmsberg 249

Hólmur 113, 144, 193, 209, 226, 245 , 255-259, 280, 281, 334, 336, 342, 351, $352,353,361,362,422,423,428,431$, 437, 446, 449, 457, 473, 475, 478-481, 483-486

Holste, Helmeke 457

Holste, Henrick 209, 480

Holste, Jochim 289, 430, 458

Holstein 50, 88, 94, 112, 114, 116, 118, 119, 126, 248

Holt 202, 235, 236
Holtgreve, Hans $177,255,314,360,430$, 432, 434, 438, 458

Holtgreve, Hinrich 314

Holtgreve, Johan 189, 248, 286, 287, 289 , 315

Holthusen, Paul 172

Honne, Hans 227, 270, 278, 480

Hooksiel 117, 273, 332, 340, 422, 479

Hornafjördur 113, 294, 298, 299, 304, 305, 424, 426, 429, 431, 434, 452

Horneman, Hinrick 187

Horneman, Marten 146, 248, 297

Horsten, Herman van 291

Housa Voe 219

Hoveman, Evert 276, 278, 325, 480

Hoya 32

Hrútafjörður 45, 144, 147, 288, 289, 430, 434, 451, 476

Hude, Hans van der 458

Hudeman, Johan 135, 263, 264, 419, 427, 430, 436, 480

Hudeman, Wilcken 262, 263, 480

Huge, Hans 176, 266, 269

Hull 70, 74, 79, 247

Hummelsen, Roleff 296

Húnaflói 288, 289

Húnavatnsping 178, 183

Húsavík 47, 177, 180, 199, 209, 288, 290-294, 320, 420, 452, 457, 462

Husman, Reineke 143

Hutlen, Hans van 249, 250, 425, 428, 431, 433, 435, 436, 438, 458

Hutlen, Lucia van 367, 455

Hutt, Peter 423

Hvaleyrargrandi 253

Hvalfjörður 121, 144, 183, 189, 214, 228 , $236,256,259,260,438$

Hylken, Johan 36

Hynrycksen, Jochim 457

Iberian peninsula 42, 321, 347, 354

Iceland 1-19, 26, 27, 30-33, 35, 36, 38, 39, 41-50, 52-59, 62-64, 69, 70-95, 97-107, 109-113, 115-125, 129, 131-133, 135, 138-149, 151-153, 155, 158-183, 185-194, 197-201, 203, 207, 208, 210, 211, 213, 214, 221, 224, 226-228, 232, 
234, 236-240, 242, 244-246, 248,

252-255, 259, 260, 264, 265, 273, 277,

280, 284-288, 290-295, 298, 301-304,

308-328, 332, 334-370, 373-375,

377-383, 421-423, 427, 443, 446-448, 450, 451, 453, 458, 466, 467, 469,

472-474, 476, 478, 479, 481, 486

Icken, Oltman 490

Ingemand, Søren 435

Ísafjarðardjúp 282, 285, 286

Ísafjarðarsýsla 183, 203, 209, 282, 285-287

Ísafjördur 110, 112, 113, 119, 283-290, 341,

$352,368,451,452,459,465,470,471$

Iserhod, Jacob 458

Italy 354

Ivenn, Marcus 459

Jacob de bartscherer 176

Jacobsen, Claus 119

Jacobsen, Jacob 358

Jacobsen, Jurgen 367, 458

Jakobsen, Jakob 441

James III, King of Scotland 84

Jaspers, Hans 177, 345, 430, 432, 434, 458

Jena 320

Jenidmen, Iven 43

Jever 117

Johan de balberer 177

Johansen, Conradt 179, 183, 287, 337, 436, 459

John Adolf, Archbishop of Bremen 270, 433

John Adolf, Duke of Holstein-Gottorp 116

John, King of Denmark 73, 94

John VII, Count of Oldenburg 32, 117, 269, 332, 340, 341, 423, 434, 437

Jökulsá 303

Jón Arason 100, 102, 162, 199

Jón Eggertson 179

Jón Egilsson 247

Jón Hallsson 198

Jón Jónsson 148, 188, 189, 193, 267, 287, 289

Jude, Andres 128, 264, 350, 418, 440

Junge, Kersten 269

Jutland 144
Kalbrandt, Claus 459

Kampen 88, 132

Kattegat 85, 144

Kaupstadartangi 271, 272

Keflavík 43, 109, 111, 113, 117, 141, 177, 209, 214, 215, 236, 244-253, 256, 345, 349, $370,375,419,422,425,428,431,433$, 436, 444, 445, 448, 450, 457, 458, 462, 464, 466, 468, 471, 474-476, 481, 485

Keiser, Frans 321

Kellinckhusen, Clawes 459

Kemmer, Hans 338

Kenckel, Detmar 263, 368, 374, 480

Kenckel, Dietrich 264, 374

Ketzeler, Herman 352

Kiel 112, 118, 341

King's Lynn 50, 70, 312

Kirkjubøur 201, 202

Kirkjufell 268

Kjósarsýsla 261

Kleve, Claus von 146, 284, 436, 459

Kleve, Hans von 135, 179, 284, 286, 341, 424, 429, 431, 436, 459

Kleve, Margaretha von 179, 459

Kloeting, Johan 144

Kloppenborg, Harmen 227, 275, 278, 344, 437

Klott, Peter 105

Knightson, Thomas 226

Knippe, Johan 480

Kock, Clawes 274, 328, 333

Kock, Hans 481

Kock, Luder 209, 244, 481

Koep, Michael 459

Kolbeinsá 290

Kolbeinsárós 290

Kolding 101, 422, 429, 432, 433, 440

Kollafjørður 181

Kolling, Joachim 117, 119, 273, 274, 276, 332, 333, 340, 341, 353, 367, 368, 422, 479

Konge, Hynrick 321

Koninck, Jorgen 327

Kópavogur 173

Kopken, Gerth 350 
Kopman, Hans 346, 364, 460

Kopman, Heinrich 260, 460

Kopman, Herman 252, 314, 433, 435, 438, 460

Koppen, Elisabeth 128,164

Koppen, Thomas 4, 98, 119, 126-129, 132, 149, 164, 180, 194, 202, 203, 336, 345, $350,368,373,378,440$

Korffmacher, Heinrich 481

Korner, Peter 175, 176, 198, 255, 371, 460

Koster, Albert 481

Koster, Friedrich 430, 481

Koster, Hinrick 490

Koster, Jacob 135

Koster, Johan 276, 277, 341, 481

Kote, Albert 278, 433, 436, 481

Krambatangi 221, 233

Krechting, Herman 144, 244, 249, 374, 375, 481

Kremer, Hans 460

Kreye, Samuel 321

Kroene, Cordt 327

Kroge, Hinrick van 373

Kröger, Johan 177

Kron, Henrick 209, 258, 327

Kronborg 422-425, 440

Kruse, Ewalt 121

Kruse, Lauritz 342

Krýsuvík 46, 244, 251, 253

Kules, Hinrik 173

Kumbaravogur 9, 55, 56, 58, 111, 113, 117, 119, 144, 149, 159, 160, 193, 199, 209, 211, 212, 223, 225, 228, 229, 242, 263 , 267-280, 332, 338, 340, 363, 368, 418, 419, 422, 423, 434, 437, 478, 479, 482-484, 486

Kummertho, Hinrick 132, 490

Kumbaravogur (Svínanes) 161, 279

Kuning, Dirick 231

Kydt, Jørgen 129, 130, 242, 243, 354, 422, 440

Ladiges, Hinrick 490

Lammers, Gise 338

Lampe, Jacob 172, 179
Lampe, Matz 128, 440

Landey 229, 270, 271, 275, 277, 432, 478, 479

Langanes 199, 294, 295, 305, 427, 430, 432, 434, 435

Langanes (Vestfirdir) 286

Lange, Jochim 177

Lange, Samuel 341

Langeyri 285

Langwedel, Carsten 367, 460

Lanthrede, Johan 481

Lapland 223

Laxfirth 134, 150, 165, 184, 216, 218, 229 , 230, 487, 489, 492

Leddinghusen, Frederik 343, 344, 358

Lee, Thomas 75, 244

Lehe, Hinrich von 481

Leiden 13

Leith 57

Lerwick 8, 218

Levenwick 219

Leyel, Frederik 281

Lindeman, Cilie 294, 297, 366, 369, 461

Lindeman, Hans 294, 297, 369, 430, 461

Lindeman, Paul 46, 52, 115, 135, 294, 296, $297,366,369,424,426,428,461$

Lindesnes 92

Lininck, Berndt 265, 346, 461

Liudt, Claus 441

Loch, Peter 134

Loen, Bernardus 321

Loen, Ditmer 461

Loen, Hans 461

Loen, Hinrick 461

Loes, Hans 181

Lofoten 37, 64, 69

Loitz, family 47, 104-106, 111, 373, 378

Loitz, Hans III the Elder 105

Loitz, Hans IV the Younger 105

Loitz, Simon 105

Loitz, Stefan 47, 54, 104-106, 224, 293, 348

Lón 303, 429

London 7, 30, 50, 68, 70, 72, 75, 89, 143, $145,191,312,313,316,347,358$

Lootsman, Jacob 219 
Loring, Ambrosius 265, 341, 345, 364, 368, 424, 426, 428, 430, 436, 462

Losekanne, Bernd 111, 114, 158-160, 189, 224, 296, 298, 300-302, 304, 334, 339, $340,356,362,363,366,376,420,421$, 482

Losekanne, Johan 304, 366, 482

Losekanne, Luder 366, 375, 482

Losekanne, Marten 302, 303, 366, 427, 429, $430,433,482$

Lubbe, Johan 482

Lubbeke, Hans van 56, 175, 198, 241, 360, 462

Lübeck $3,4,7,11,15,18,22,30,33,40,47$, $49,55,58,66,68,72,75-78,80,81$, $83-85,87,90-93,95,97,98,100-102$, 104, 106, 110-113, 123, 127, 135, 144, $146,147,155,171,172,175,180,188$, 202, 209, 225, 241, 242, 255, 258, 259, 273, 279, 281, 283, 284, 286, 290, 292, 296, 297, 327, 334, 337, 342, 349-352, $354,356,358,360,367,369,374,377$, 378, 382, 417-419, 422, 423, 429, 431, 435, 437, 438, 449, 478, 484

Lude, Claus 203, 205, 245, 420, 482

Luders, Hans 462

Luders, Johan 482

Luers, Carsten 490

Luers, Hans 462

Luessen, Hans 462

Luessen, Hinrich 462

Lumisdale, Jhone 153

Lunda Wick 2, 200, 203, 488, 491

Lüneburg 31, 41, 87, 90, 112

Lunenberg, Cordt 209, 241

Lunge, Vincent 126

Lunsman, Herman 490

Luse, Dirick 490

Lutke, Jacob 472

Magdeburg 31

Magnus IV, King of Norway 66

Magnus, Olaus $11,13,26,27,32,34,45,47$, $49,51,74,152,156,208,222,223$

Mainland 218, 220
Mainz, Electorate of 28

Mair, Edward 153

Make, Hans 462

Malmö 120, 122, 146, 183, 354, 418, 476

Man, Isle of $63,135,358$

Margaret of Denmark, Queen of Scotland 84

Maríuhöfn 260

Marteinn Einarsson 100

Martens, Hinrick 144, 198, 462

Mary Stuart, Queen of Scots 133, 487, 488, 489, 492

Matthiasen, Lazarus 178

Matyes de Barscher 176

Matzen, Oluf 130, 336, 348, 440, 441

Meinsen, Joachim 353

Melrakkaslétta 293, 294

Melsow, Gerd 267, 438

Meyer, Bartold 490

Meyer, Carsten 343

Meyer, Christoffer 111, 114, 164, 177, 224 , 226, 277, 296, 301, 302, 334, 335, 340, 341, 356, 357, 361, 362, 366, 376, 421, 483, 490

Meyer, Cordt 166, 490

Meyer, Dirick 462

Meyer, Gerdt 98

Meyer, Gyse 463

Meyer, Hans 176, 197, 219

Meyer, Henninck 176

Meyer, Herman 153

Meyer, Hilmer 134, 490

Meyer, Hinrick 209, 269

Meyer, Jacob 490

Meyer, Luder 366, 483

Meyer, Lutke 462

Meyer, Marx 98

Meyer, Wernerus 321

Michel, Johan 134, 216, 490

Middendorp, Herman 491

Mildehouet, Hinrich 463

Minden, Asmus van 191

Minden, Cordt van 191, 463

Minden, Didrick van 75, 99, 191, 244, 255

Minden, Hinrich van 491

Moige, Bartold 483 
Moldenhouwer, Hans 177

Moller, Bartelt 338, 347, 348

Moller, Christoffer 368

Moller, Cordt 364, 435, 438, 463

Moller, Dirick 373, 463

Moller, Hans 463, 464

Moller, Herman 464

Moller, Hinrich 199, 295, 297

Moller, Jochim 464

Moller, Jurgen 464

Molre, Marten 176

Monnickhusen, Clawes 9, 18, 31, 33, 52, 55, $56,58,59,138,159,160,170,171,199$, 209, 212, 227, 273, 343, 363, 483

Morton, William, Earl of 40

Mot, Andrew 134

Mowat, Gilbert 53, 165

Mowat, James 316

Moyde, Dirick thor 464

Múlasýsla 188, 189, 199, 212, 296, 298, 305

Múlaping 181, 192

Mule, Lorentz 101, 102, 168, 171, 187, 226, 236, 239, 255, 260

Mumme, Augustin 50

Mumme, Heinrich 111, 300, 419

Mumme, Johan 179

Mumme, Marcus 50

Münster 145

Munsterman, Hinrick 483

Munsterman, Johan 117, 119, 144, 146, 173, $225,272-274,276,279,338,344,368$, 419, 483, 485

Mutenhusen, Bastian 177

Mutter, Tonnies 327, 417

Mýrar 192

Mývatn 46, 188, 244, 288, 292, 293

Nagel, Bruning 118, 277, 341, 484

Nagel, Clawes 491

Nanneke, Hans 464

Naries, Johan 464

Nebebak, Orne 184, 335

Nekel, Henrich 464

Nesting 164
Nesvogur 44, 113, 116, 118, 139, 176, 193, 227, 267, 269, 270, 272-280, 338, 341, 423, 432, 434, 437, 478, 480-486

Netherlands 40, 44, 85, 97, 100, 123, 125 , $129,133,200,221,225,233,248,253$, 292, 299, 354, 375

Newfoundland 41

Neygenborch, Hans 465

Neygenborch, Marten 465

Nidingen 85, 144

Niebeke, Clawes 465

Nielsen, Hans 290, 350, 418

Nielsen, Joen 128, 194

Nielsen, Mogens 441

Nielsen, Søren 441

Nilsson, Olaf 81

Niport, Anthony 135

North America 80

North Sea 52, 61, 63, 64, 66, 68, 72, 83, 124, 133, 141, 330, 383

Northmavine 53, 164, 196, 221

Norway 3, 5, 9, 22, 35, 36, 37, 38, 39, 41, 47, 57-59, 61-69, 71, 73, 77-79, 81, 85, 87, 90-92, 94, 97, 98, 124-129, 131-133, $143,149,154-158,161,164,166,183$, $185,190,195,197,208,210,233,253$, $260,292,316,354,357,377,379,445$

Novgorod 68, 89, 327

Nyborg 418, 420

Nyling, Diderich 465

Nynberch, Detmer 465

Oddeyri 292

Odense 92, 93, 440

Ögmundur Pálsson 99, 100, 174, 175, 178, 198

Ólafsvík 211, 266, 273

Olde, Claus 297

Oldehorst, Albert 465

Oldenburg $7,9,13,16,18,22,43,44,52,55$, $56,58,59,115,119,139,159,160,170$, 171, 193, 199, 212, 227, 267, 270, 271, 272, 274, 275, 277, 278, 328, 332-334, $337,340,343-346,353,360,362,367$, 368, 374, 423, 434, 437, 479

Oldenburg, Brun 491 
Oldenburg, County $32,98,117,118,120$, 193, 273, 274, 277, 432

Oldenburg, Hinrick 491

Oldenbuttel, Johan 189, 303, 433, 437, 484

Oldensche, Herman 258, 352, 484

Oldenseel, Herman 111, 135, 258, 296, 300, $327,350-352,358,418,484$

Oldesloe 119, 293

Ölfusá 240, 242

Olrickes, Hans 177

Olrickes, Olrick 465

Olrickes, Reinholt 179

Oltken, Jurgen 328

Önundarfjörður 202, 235, 236

Øresund 72, 93, 100, 106, 118, 123, 352, 353,440

Örfirisey 254, 257

Óseyri 234

Orkney $6,8,40,41,52,69,78,79,82-84$, $131,135,143,155,184$

Orkney, Diocese 79, 197

Orkney, Earldom 62, 131, 133, 195

Oslo 69,78

Osnabrück 145

Osnabrugge, Hans van 366, 484

Osnabrugge, Hinrick van 366, 484

Osthoff, Bernd 245, 345, 425, 426, 428, 433, 437, 465

Ostrabord 222

Otken, Claes 176

Ottersen, Luder 4, 76, 143, 180, 226, 242, 243, 258, 259, 274, 281, 284, 297, 327, $337,342,350-355,382,422,428,429$, 431, 435, 437, 438, 449, 478

Páll Jónsson 183, 280, 423, 427, 480

Papa Stour 164, 196, 216, 218, 219, 221

Papey 212, 299

Patreksfjörður 109, 146, 215, 281-284, 286, 418, 422, 459

Pedersen, Knut 248, 418

Pedersen, Rasmus 291, 292

Peerse, Gories 1-3, 11-16, 39, 139, 142, 151, 172, 177

Pennycuke, John 491
Perlmeyer, Pawel 176

Pestorp, Dirick 491

Petersen, Peter 321

Pétursson, Tyl 187

Pineman, Dirick 43, 177

Pining, Didrick 73, 74, 94, 185, 190, 191

Piper, John 134

Poek, Henrik 175

Poland 154

Polmeyer, Pauwel 177

Pöner, Fritz 252

Pöner, Hans 435

Pöner, Rotman 119, 252, 433, 435, 438

Pool of Virkie 219, 220

Portugal 13, 42, 359

Potthorst, Hans 190, 191

Pren, Harmen 465

Priester, Niclas 125, 439

Pundt, Hinrick 484

Qwast, Marcus 179

Rademan, Claus 423

Ramke, Hinrich 465

Rangárping 188

Rantzau, Gert 50

Rapnes 184

Rathman, Henninck 466

Ratke, Heine 273, 278, 338, 376, 484, 485

Ratkens, Hinrich 151, 357, 435, 466

Ratkens, Reimer 248, 431, 433, 437, 466

Raufarhöfn 294

Recken, Hans 466

Red Sea 179

Rede, Johan van 484

Reimers, Brun 362, 375, 484, 491

Reineke, Johan 303, 334, 433, 484

Reiners, Johan 144, 491

Reinicke, Herman 180

Reinstorp, Jurgen 251

Reise, Hieronymus 347 
Rendsburg 118, 119, 252, 433, 435

Reykhólar 202, 204

Reykjanes 38, 39, 213, 214, 236, 237, 239, 240, 243, 244, 246, 249, 253, 256, 361, $370,381,453$

Reykjarfjörður 289

Reykjavík 209, 236, 256, 257, 259, 260

Reymels, Clawes 484

Reyðarfjörður 296, 298, 301, 302, 304, 447, 451, 452, 464, 471

Rhine 30

Rhineland 55

Rieke, Simon 466

Ríf $45,72,109,113,135,159,173,176,177$, 209, 211, 262, 264-267, 269, 272, 273, 280, 341, 355, 371, 418, 421, 422, 424, 426-429, 438, 444, 446, 448, 451, 454-457, 465, 467, 469, 471, 473, 476,486

Riga 327

Ripen, Andres van 345

Rocks, Peter 153

Rode, Claus 106, 209, 292, 327

Rode, Tonnies 438

Rodewolt, Lutke 177

Roegerinck, Herman 466

Roen, John 134

Rogge, Wilken 466

Rolfs, Hans 106

Rome 11

Ronne, Hinrick van 74, 292

Ropsleger, Ropke 484

Roremberg, Johannes 45, 46, 53

Rors, Hans 466

Rosenbrock, Alheyd 368

Rosenbrock, Dithmer 368

Rosenbrock, Hans 368

Roskilde 420, 440

Rostock 66, 87, 174, 175, 342, 349, 350

Rotmert by dem bartscher 176

Rowolt, Hans 466

Rulves, Brüning 325, 363

Rulves, Heinrich 119

Rumpolt, Marx 28

Runge, Johan 215, 216, 361, 488, 491
Russe, Bernard 135

Russia 321, 354, 359, 457, 474

Ruther, Gerdt 327

Rye 441

Rykelman, Peter 365, 366, 466

Sabel, David 466

Sabel, Herman 466

Sæmundur Magnússon Hólm 268

Salefeld, Bernd 143, 173, 267, 280, 296, $336,355,356,360,424,426-429,438$, 467

Salefeld, David 267

Saler, Hans 177

Salomon, Hinrick 117, 276, 338, 344, 374, 485

Salthen, Jacob van 327

Salzwedel 145

Sand 153

Sandemann, Hans 43

Sander, Heine 198, 467

Sandoy 180

Sandsting 164, 184

Sasse, Hinrich 199

Scalloway 134, 153, 196, 197, 200, 216, 380, 490

Scandinavia 27, 28, 55, 61, 63, 98, 124, 152, $155,156,188,232,315$

Scania $120,168,178,179,310,311,315,327$, $346,347,373,381,443$

Schapeskop, Jacob 342, 467

Scheidewint, Adam 101

Schinckel, Jurgen 248, 364, 429, 467

Schleswig, Duchy 94, 114, 116, 119

Schmid, Helmich 327, 417

Schmidt, Brandt 342

Schmidt, Hinrick 177, 438

Schmidt, Lutke 75, 101, 140-142, 171, 247, 312

Schmidt, Simon 302

Schneman, Herman 164, 165, 184, 492

Schneman, Tonnies 164, 165, 226, 492

Schoff, Clawes 341

Schomaker, Frantz 491 
Schomaker, Hans 105, 144, 266, 294, 345, 346, 427, 430, 468

Schomaker, Herman 244, 249, 374, 375, 485

Schonefeld, Johan 321

Schrevenhof 175

Schroder, Evert 135, 485

Schroder, Godert 373, 468

Schroder, Henrick 57, 491

Schroder, Herman 134, 491

Schroder, Johan 251, 428, 485

Schroder, Laurens 289, 430, 443, 468

Schulle, Johan 216, 335, 491

Schulte, Asmus 54, 443, 468

Schulte, Gert 485

Schuren, Herman van 255, 338, 360

Schutte, Hinrick 251

Schuweshusen, Hans 469

Scollay, Robert 184

Scotland 5, 6, 8, 17, 40-42, 49, 57, 63, 76, $84,124,131-133,135,136,143,175$, 184, 195, 219, 220, 232, 294, 316, 357, $359,378,379,467,490$

Segeberg 75, 110, 118, 119, 186, 271, 275, 286, 348, 419, 420, 437

Segelcken, Hinrick 203, 491

Segelcken, Roloff 491

Seller, John 229, 230

Selman, Andreas 292, 437, 469

Seltjarnarnes 256, 257

Setberg 199

Shetland 1-6, 8, 9, 11, 15, 17-19, 23, 27, 28, $35-37,40-44,48,49,52,53,57,59$, $62,69,78-80,82-84,87,88,90,120$, $124,128,131-136,140-144,148,150$, 153, 155, 164-166, 173-175, 180, 184, 195-197, 200, 203, 207, 208, 215-221, 224-226, 229, 230, 232, 233, 236, 316, $321,323,324-326,335,336,342,343$, 347, 356-358, 360-362, 367, 370, 371, 375-383, 443, 447, 448, 451, 453, 454, 456, 458-461, 463, 467, 474, 475, 487-492

Sigurd hialtt 180

Sillem, Anna 473

Simessen, William 134
Sinclair, George 184

Sinclair, Matthew 184

Sinclair of Aith, Arthur 196

Sinclair of Havera, Olave 195, 215, 216, 218-220

Sivers, Albert 199, 291, 367, 432, 436

Sivers, Peter 45, 108, 258, 289, 369, 429, 431, 436

Skagafjördur 144, 199, 209, 290, 291, 302, $366,367,420,458,464,476$

Skagaströnd 108, 113, 146, 189, 248, 258, 288-291, 338, 369, 426, 429, 431, 433, 436, 447, 450, 454, 458, 468, 469, 472, 473

Skálholt 56, 71, 99, 100, 172, 174, 175, 191, 197, 198, 200, 239, 240, 241, 253, 254

Skanderborg 422, 423, 433

Skelda Voe 218

Skeldberrie 165

Skinkel, Niels 149, 440

Skriver, Christian 102

Skriver, Mikkel 128, 440

Skriðuklaustur 181, 182, 192, 475

Skutulsfjördur 109, 209, 215, 285, 286, 422, 425, 428, 431, 433

Slochter, Johan 485

Sluter, Hinrick 279, 327, 422

Smidt, Hans 177, 209, 360

Smith, Andrew 185

Snæfellsjökull 263, 266

Snæfellsnes 38, 109, 148, 160, 192, 193 , 203, 212, 214, 229, 239, 261, 263, 265, $267,271,275,289,361,380,381,478$, 481

Snepel, Paul 50

Sound of Papa 216, 218

Spain 42, 55, 359

Spákonufellshöfði 288

Sparke, Heinrich 78, 79

Speyer 357

Sprenger, Christoffer 492

Sprenger, Hinrick 164, 226, 335, 357, 361, 492

Stade $7,112-114,133,149,189,283,284$, $286,287,316,360,421$ 
Staeffhorst, Meineke 485

Stael, Cordt 241, 327

Stakksfjördur 249

Stall, Asmus 264

Stavanger 92, 99

Steding, Carsten 375, 492

Steffens, Frantz 485

Steinkamp, Hans 245, 246, 345, 431, 433, 437

Stemmelman, Anna 451

Stemmelman, Christina 367, 458

Stemmelman, Jochim 470

Sten, Cordt $80,171,327$

Sten, Henning 80, 327

Sten, Lutke 254

Stene, Marten 84, 144, 343, 344, 358

Stettin 47, 104, 348, 378

Stewart, Robert, Earl of Orkney 134, 196, 197, 226

Stigsen, Otto 101, 151-153, 161, 168, 187

Stigsen, Paul 50, 104, 178

Stirling, William 184

Stockholm 327

Stralsund 66, 87, 350

Strandasýsla 178, 183, 288

Straumfjörður 251

Straumur 113, 118, 119, 236, 244, 247, 250-253, 256, 422, 433, 435, 438, 455, $458,460,474$

Streymoy 183, 201

Stroteman, Reineke 369, 485

Struckman, Henning 106

Struckmeyer, Herman 177, 209, 255, 266

Stubbe, Dirick 344

Stuue, Nicolaus 321

Stykkishólmur 18, 115, 193, 269, 272, 275 , 276, 278, 361, 433, 436, 477, 479, 481, 482

Sumburgh Head 134, 218, 219, 490

Surbeck, Cordt 180

Surbeck, Simon 76, 180, 238, 354

Surman, Jacob 219

Suduroy 221, 233

Sveinn Simonarson 203

Sverre, King of Norway 62

Svínanes 161, 279, 282

Swabia 29
Swachman, Reineke 485

Swake, Hans 128

Swarte, Clawes 327

Sweden 33, 55, 63, 93, 97, 104, 105, 109-112, 125, 133, 349, 369, 445

Swer, Laurens 177

Sydenborch, Hans 176, 181, 209

Symbister 229, 231

Tacke, Cordt 287, 345, 425, 428

Tálknafjörður 109, 215, 282, 283, 418

Tappe, Hans 199, 247, 269

Teieman, Luder 492

Temmerman, Hans 177, 425, 471

Terminau, Joachim 267, 356

Theeten, Hans 471

Thehus, Hans 194

Thim, Joachim 44, 111, 129, 130, 144, 183 , 195, 249, 253, 345, 349, 360, 361, 419, 440, 471

Thim, Jurgen 341, 479

Thode, Hans 471

Thode, Jacob 162, 472

Thomsen, Heinrich 423

Thuringia 29

Tideman, Lubberd 268

Tilebare, Friedrich 192, 303-305, 429, 437, 485

Tilebare, Gerdt 369, 485

Timmerman, Ratke 108, 189, 289, 291, 426, 472

Tinappel, Bartholomeus 92, 110, 119, 162, 284, 286, 287, 327, 328, 349, 369, 374, 418

Tinappel, Margaretha $110,162,283,286$, 369, 419

Tinganes 149, 221, 233

Tingwall 165, 196

Tinsdal, Hans 296, 338, 472

Tinsdal, Johan 297

Tønsberg 69, 78

Torfnes 302

Tórshavn 149, 221, 233

Trolle, Birge 111, 263, 272, 276, 418

Trolle, Herluf 104

Trondheim 92, 127

Trongisvágsfjörður 221 
Trupe, Hinrich 485

Tulloch, David 166, 490

Tulloch, Lourence 165

Ulenhot, Remmert 80, 327

Unst 1, 2, 164, 165, 200, 203, 215, 216, 221, 225, 229-231

Uppsala 11

Ur, John 134

Urafirth 153

Utrecht 85

Uyeasound 134, 153, 164, 165, 215, 216, 229 , 230, 487-489

Vaget, Hinrick 187, 492

Vaila Sound 216

Valckendorf, Christoffer 114, 129, 130, 284, 286, 291, 369

Valeman, Joachim 173, 255, 338, 425, 472

Varel, Gerdt van 333, 344

Varenhorst, Herman 177

Vasmer, Catharina 463

Vasmer, Dirick 274, 276, 277, 296, 472, 485

Vasmer, Johan 485

Vatnajökull 303

Vatneyri 284, 424, 429, 431, 436

Vatnsleysa 119, 148, 236, 247, 250-252, $256,422,428,433,435,438,455,458$, $460,474,485$

Vebbeke, Cordt 241, 327

Veckinghusen, family 329

Veere 125

Velde, Paul vam 90

Venice 11

Verden 264, 374, 480

Vesterålen 69

Vestmannaeyjar 38, 76, 103, 180, 223, 237, $238,239,350,354,417,476$

Vibbe, Christen 435

Vibbe, Mikkel 435

Vicke, Peter 177

Vigfús Erlendsson 159, 163, 188

Vigfús Porsteinnsson 189, 295

Vilter, Jurgen 289, 338, 369, 433, 436, 473

Visby 110, 286

Viðareiði 203

Viðey 99, 191, 199, 319
Viðoy 203

Vlenhop, Matthias 251

Voege, Dirick 134, 153, 165, 215, 489

Vögeler, Marten 473

Vogit, Johan 281, 353, 435

Vogler, Christof 110, 119, 286, 348, 419, 420

Volmers, Berwardt 492

Vopnafjörður 111, 113, 115, 135, 159, 188, 192, 211, 258, 294-300, 302-304, 342, $346,350,351,353,360,366,369,418$, 421, 424, 426, 428, 430, 432, 435, 438, 444, 446, 447, 452, 456, 457, 460, 461, 468, 472, 475

Vos, Hans 353

Vriensteen, Dideric 84

Vriese, Frederyck de 125, 126, 439

Vurborn, Herman 255, 327, 417

Vynland 80

Wake, Reiner 486

Walleman, Cordt 436, 486

Walleman, Dirick 275

Walls 216, 218

Warder, Baltzer vam 365, 473

Warder, Joachim vam 365, 473

Warder, Marten vam 473

Wardis, Margaret 165

Warmsen, Clawes 473

Warneke, Cordt 184

Warneke, Joachim 294, 297, 302, 304

Warners, Johan 451

Wedeman, Herman 209, 301, 304, 325, 334 , 366, 486

Wedeman, Luder 334, 362, 366, 486

Wederbar, Richard 135, 264, 265, 267, 421

Wegener, Andreas 243, 246, 474

Wegener, Hans 239

Wegener, Herman 242, 243, 352, 425, 474

Wegener, Jurgen 265, 474

Wegener, Marten 243

Weinreich, Casper 85

Wemeyer, Cordt 252, 433, 435, 474

Wencke, Jacob 176

Wendelcke, Johan 441

Werenberch, Johan 486

Werenborg, Johan 344

Weser 32, 111, 144, 272, 324, 375 
Westerwold, Gerdt 361, 486, 492

Westfalia 66

Westfjords 36, 38, 173, 179, 183, 202, 214, 215, 236, 281-285, 287, 381, 453

Westphall, Joachim 474

Whalsay 134, 150, 216, 218, 229, 231, 487, 489, 491

Wicherling, Gerdt 492

Wichman, Hinrich 347

Wichman, Joachim 111, 130, 131, 163, 195, $253,336,338,345,347-349,360,361$, 419, 440, 441, 475

Wichman, Peter 347

Wickboldt, Ladewich 324, 335, 492

Widen, Lambert tor 486

Wiggers, Johan 486

Wilckens, Alert 492

Wilde, Hinrick 374

Wilkens, Herman 437, 486

Willer, John 292

Willers, Joachim 209

Willers, Oldtman 492

Wilster 118, 248, 423

Winckelman, Laurens 342

Winock, Jacob 192, 194, 295, 297, 305, 432, 435, 475

Winsen, Hans van 476

Winsen, Jurgen van $289,434,476$

Winterlandt, Jurgen van 475

Winters, Reineke 334, 369, 486

Wirckes, Peter 342

Wismar 87, 113

Wispendorf, Ludke 85, 144

Wisseloh, Wend 486

Witstock, Hermen 155, 180

Witte, Henrick 209, 290

Witte, Peter 177

Wittemutze, Claus 144

Wittenberg 29, 174

Wittenborch, Dyryck 475

Wittenborch, Helmeke 346, 347, 367, 460, 475
Wittenborch, Hille 367

Wittesant, Clawes 486

Witting, Hinrich 354

Wittorp, Hinrick 475

Wolders, Peter 476

Woltken, Herman 476

Wordenhoff, Jasper 476

Wulffers, Johan 492

Wullenwever, Joachim 97, 125, 126, 127, 128, 181, 194, 197, 202, 239, 350, 373, 417, 439, 476

Wullenwever, Jurgen 127

Wullenwever, Oleff 181

Wulveken, Mattheus 341

Wunderlick, Jurgen 321

Yell 48, 164, 165, 184, 216, 221

York 30

Yorkshire 143, 191

Zerneman, Tyleman 111, 263, 272

Zimmerman, Moritz 358

Zuiderzee 66,68

Pingeyrarklaustur 183

Pingeyrarping 295

Pingeyri 285

Pingvellir 185, 186, 239, 254

Pistilfjördur 294

Pjórsá 240

Porlákshöfn 113, 209, 240, 242, 243, 419, 420, 422, 425, 429, 474

Porleifur Björnsson 190

Porleifur, son of Cordt Surbeck 180

Pórshöfn (Reykjanes) 246-248, 423

Pórshöfn (Langanes) 119, 189, 192, 199, 294, 295, 297, 298, 304, 345, 346, 360, 369, 427, 430, 432, 435, 438, 444, 452, 457, 468

Pykkvabær 192, 203, 445

Pýskuvör 279 